\title{
Rapid species- and subspecies-specific level classification and identification of Lactobacillus casei group members using MALDI Biotyper combined with ClinProTools
}

\author{
Chien-Hsun Huang ${ }^{1}$ and Lina Huang \\ Bioresource Collection and Research Center, Food Industry Research and Development Institute, Hsinchu 30062, Taiwan
}

\begin{abstract}
Using common taxonomic methods such as 16S rDNA sequencing and physiological and biochemical analysis to identify members of the Lactobacillus casei group (LCG) is time-consuming, expensive and inaccurate. In this study, we applied matrix-assisted laser desorption/ ionization time-of-flight mass spectrometry (MALDITOF MS) to rapid discriminate LCG strains by creating an analytical in-house database (IHDB) and to develop a classification model for subspecies-level differentiation based on MS biomarkers using ClinProTools bioinformatics software (Bruker Daltonics, Billerica, MA). Genotypic methods (housekeeping gene sequencing and species-specific PCR) were also established to validate the MALDI-TOF MS platform. A total of 48 LCG reference strains were correctly identified at the species level (mean score: $2.45 \pm 0.1$ ) by using MALDI-TOF MS with an IHDB and had high score values, which was in accordance with results from mutL gene sequencing and specific PCR-based methods. However, one strain that was identified as $L$. casei had a relatively low score value $(2.02 \pm 0.02)$, lower sequence similarities (mutL: $90.4 \%$ ), and failed to amplify a species-specific amplicon; it may therefore represent an undescribed novel species. In addition, after implementation of the classification model (based on 2 biomarker peaks: $\mathrm{m} / \mathrm{z}$ 4,930 and 5,303), L. paracasei strains could be clearly and easily differentiated to the subspecies level. Afterward, 7 LCG-related isolates from different probiotic samples were analyzed and accurately identified. Our data demonstrate the high-resolution performance of MALDI-TOF MS for fast and accurate demarcation of LCG strains when used with an IHDB coupled to ClinProTools; this methodology can serve as an alternative for quality control of probiotic products.
\end{abstract}

Received August 7, 2017.

Accepted September 30, 2017.

${ }^{1}$ Corresponding author: chh@firdi.org.tw
Key words: MALDI-TOF MS, ClinProTools, DNA sequencing, novel candidate taxon, Lactobacillus casei group

\section{INTRODUCTION}

The nomenclature and taxonomic status of the Lactobacillus casei group (LCG) have been controversial for a long time (Skerman et al., 1980; Collins et al., 1989; Dellaglio et al., 1991, 2002; Chen et al., 2000). The Judicial Commission of the International Committee on Systematic Bacteriology in 2008 concluded that the current taxonomy of the LCG still comprises 3 closely related species: Lactobacillus casei, Lactobacillus paracasei (ssp. paracasei and ssp. tolerans), and Lactobacillus rhamnosus (Tindall, 2008). The LCG members contain many strains generally recognized as safe and have been largely applied in probiotic products. Lactobacillus casei Shirota and L. rhamnosus GG are well-known commercial probiotic strains in dairy supplements and foods, and they exhibit antitumor, immunostimulatory, and antimicrobial effects (Shida and Nomoto, 2013; Ashraf and Shah, 2014; Reid, 2015; Orlando et al., 2016). However, more than $28 \%$ of commercial probiotic products are mislabeled at the genus or species level because of the use of methods that have limited taxonomic resolution (Huys et al., 2006). Thus, the ability to quickly and accurately identify starter cultures is important for the management and quality control of commercial probiotic products.

Researchers have traditionally and primarily relied on carbohydrate fermentation tests to differentiate the members of the LCG. However, the phenotypic characteristics that form the basis of these tests may be affected by environmental conditions or by the possible loss or acquisition of plasmids that encode many carbohydrate fermentation traits, resulting in isolates that exhibit atypical metabolic features (Ahrne et al., 1989). Deoxyribonucleic acid fingerprinting has been used for differentiation of lactobacilli, such as amplified fragment-length polymorphisms, random amplification of polymorphic DNA, repetitive sequence-based PCR, 


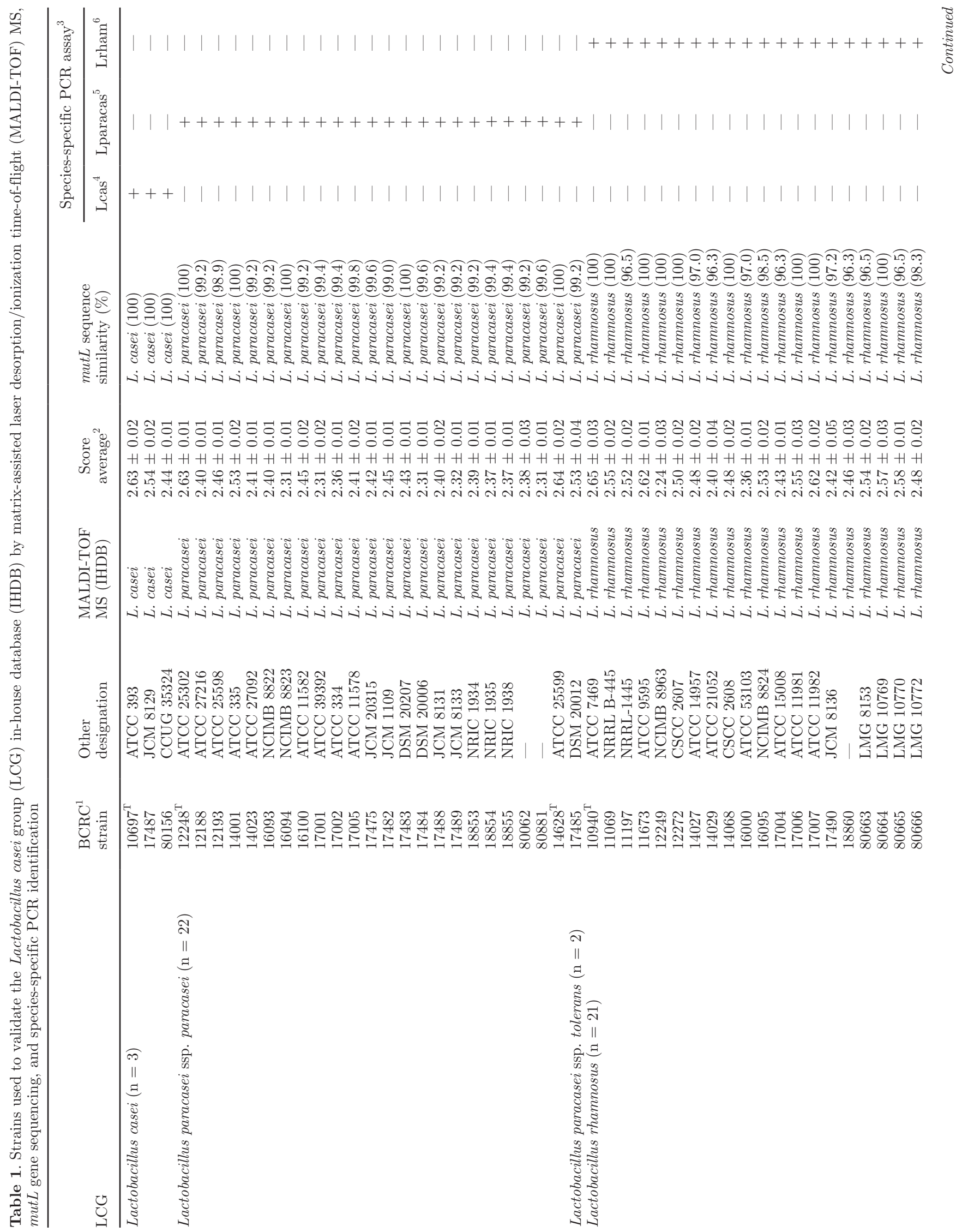


amplified rDNA restriction analysis, and intergenic spacer region-PCR (Mattarelli et al., 2014). At present, $16 \mathrm{~S}$ rRNA gene sequencing is the genotypic method that is most commonly used to assign bacterial taxonomy and phylogeny (Petti et al., 2005). The cut-off value of $16 \mathrm{~S}$ rRNA gene sequence similarity to identify an organism at the species level is approximately 98.65\% (Kim et al., 2014). Unfortunately, members of the LCG are sometimes poorly resolved, leading to misidentification at the species level due to the high genetic similarity (reaching 98.7-99.9\%) of their $16 \mathrm{~S}$ rRNA genes (Huang et al., 2015). By contrast, proteincoding genes provide greater discrimination power and can serve as an alternative to $16 \mathrm{~S}$ rRNA gene sequencing to identify members of the LCG (Huang and Lee, 2011; Huang et al., 2014, 2015). The traditional gold standard for bacterial species delineation is DNA-DNA hybridization (Stackebrandt et al., 2002), and the whole-genome sequence comparison method is becoming a new trend for determining microbial taxonomy. The average nucleotide identity cut-off value to establish a species boundary between 2 genomic data sets is approximately 95\% (Varghese et al., 2015). However, these technologies are time consuming, labor intensive, costly, and difficult to use routinely in laboratories.

Matrix-assisted laser desorption/ionization timeof-flight (MALDI-TOF) MS is a phenotype-based method that can be used for the identification, classification, and dereplication of microorganisms based on their specific protein profiles (Moore and Rossello-Mora, 2011). The advantages of MALDI-TOF MS include that it is easy to perform, fast, accurate, economical (despite the initial cost of the instrument), and high throughput (Neville et al., 2011; Sandrin et al., 2013; Tran et al., 2015). It has been successfully applied in routine clinical diagnosis, and its use has expanded to the research areas of environmental monitoring, food safety, biodiversity, and gut microbiotas (Pavlovic et al., 2013; Samb-Ba et al., 2014; Santos et al., 2016). In general, MALDI-TOF MS can discriminate at both the genus and species levels using proteomics-based identification, and the taxonomic resolution can reach the subspecies and strain levels when the method is combined with specific biomarkers (Spinali et al., 2015). However, reference databases are mainly designed for routine clinical practice (Bizzini and Greub, 2010), and adding additional entries to the database is quite important to increase identification rates via matching. In this study, we determined the performance of MALDI-TOF MS when used with an in-house database (IHDB) coupled with ClinProTools (Bruker Daltonics, Billerica, MA) to discriminate between members of the LCG; DNA-based analysis such as sequencing and species-specific PCR assays were also used as reference methods. 


\section{MATERIALS AND METHODS}

\section{Lactobacillus Strains and Culture Conditions}

All reference strains used in this study were obtained from the Bioresource Collection and Research Center (BCRC; Food Industry Research and Development Institute, Hsinchu, Taiwan) and have been authenticated by $16 \mathrm{~S}$ rRNA gene sequencing (Table 1). The Lactobacillus strains were incubated on lactobacilli de Man, Rogosa and Sharpe agar (Difco, Becton, Dickinson and Co., Franklin Lakes, NJ) anaerobically for $20 \mathrm{~h}$ at $37^{\circ} \mathrm{C}$, and fresh cultures were used for further DNA-based and proteomics-based analysis.

\section{DNA Preparation and Design of LCG Degenerate Primers}

Genomic DNA was extracted using the DNeasy kit (Qiagen, Valencia, CA) following the manufacturer's instructions. The DNA concentration and quality were calculated from the $260 / 280 \mathrm{~nm}$ absorbance ratio and checked by agarose gel electrophoresis. The degenerate primers Lcagp-mutlF3 (5'-ATTGAYCARCTATCKGCMAC-3') and Lcagp-mutlR4 (5'-GAGCCRTAACCGGCAAMA-3') were designed and targeted to the most conserved region of the mutL gene, which was determined by comparing the mutL gene from the whole-genome sequences of several LCG species (accession no. AP012544, CP000423, CP003094).

\section{DNA Sequencing of mutL Gene}

Partial fragments of the mutL gene were amplified and sequenced using consensus degenerate primers. The thermal cycler protocol consisted of the following steps: initial strand denaturation at $94^{\circ} \mathrm{C}$ for $5 \mathrm{~min}$; followed by 30 cycles of $94^{\circ} \mathrm{C}$ for $1 \mathrm{~min}, 60^{\circ} \mathrm{C}$ for $1 \mathrm{~min}$, and $72^{\circ} \mathrm{C}$ for $1.5 \mathrm{~min}$; and a final extension step at $72^{\circ} \mathrm{C}$ for 7 min. The resulting amplicons were purified using a QIA quick PCR purification kit (Qiagen) and sequenced using a BigDye Terminator version 3.1 cycle-sequencing kit and a 3730 DNA sequencer (Applied Biosystems, Foster City, CA).

\section{Phylogenetic Analysis}

All sequences were aligned using ClustalX version 1.8 (Thompson et al., 1997), and the sequence identities were calculated using MatGAT version 2.02 (Campanella et al., 2003). A phylogenetic tree was constructed by using the PHYLIP computer program package and the neighbor-joining method, with genetic distances computed using Kimura's 2-parameter model (Felsenstein, 1993). Bootstrap analyses with 1,000 replicates were used to estimate the confidence of each tree node (Kimura, 1980).

\section{Design of Species-Specific Primers and PCR-Based Identification}

The PCR oligonucleotide primer sets specific for $L$. casei, L. paracasei, and L. rhamnosus were designed using VectorNTI version 9.0 (Invitrogen, Carlsbad, CA). All LCG strains and 8 nontarget strains were selected for species-specific PCR testing (Table 1). The thermal cycler protocol consisted of the following steps: initial strand denaturation at $94^{\circ} \mathrm{C}$ for $5 \mathrm{~min}$; followed by 30 cycles of $94^{\circ} \mathrm{C}$ for $1 \mathrm{~min}, 65^{\circ} \mathrm{C}$ for $1 \mathrm{~min}$, and $72^{\circ} \mathrm{C}$ for 1 min; and a final extension step at $72^{\circ} \mathrm{C}$ for $7 \mathrm{~min}$.

\section{MALDI-TOF MS Analysis}

A standard ethanol/formic acid extraction protocol was performed by following the method described by Huang et al. (2016). One microliter of protein extract was spotted on a 96-spot polished steel MALDI target plate. After the spots were dried at room temperature, they were overlaid with $1 \mu \mathrm{L}$ of $\alpha$-cyano4-hydroxycinnamic acid matrix for sample/matrix cocrystallization. Mass data acquisition was performed using a MALDI-Microflex LT instrument spectrometer (Bruker Daltonics GmbH, Bremen, Germany) in positive linear mode (mass range: 2,000-20,000 Da). The bacterial test standard was used for instrument calibration. Moreover, the positive and negative controls were conducted using Escherichia coli BCRC 11634 and noninoculated matrix solution, respectively.

\section{LCG IHDB Creation, Validation, and Dendrogram Construction}

The raw spectra were acquired for each type strain, and the quality and relationships of each spectra were checked, screened, and estimated using FlexAnalysis software version 3.4 (Bruker Daltronics GmbH, Bremen, Germany) and a composite correlation index (CCI). The best quality spectra that remained were chosen and transferred to create a specific single main spectrum profile (MSP) for database expansion. The identities of all LCG reference strains and isolates were validated based on the newly created database. Results were analyzed following the scoring system according to the manufacturer's instructions. Samples with score values between 2.30 and 3.00 were considered to be reliably identified at the species level; samples with values 


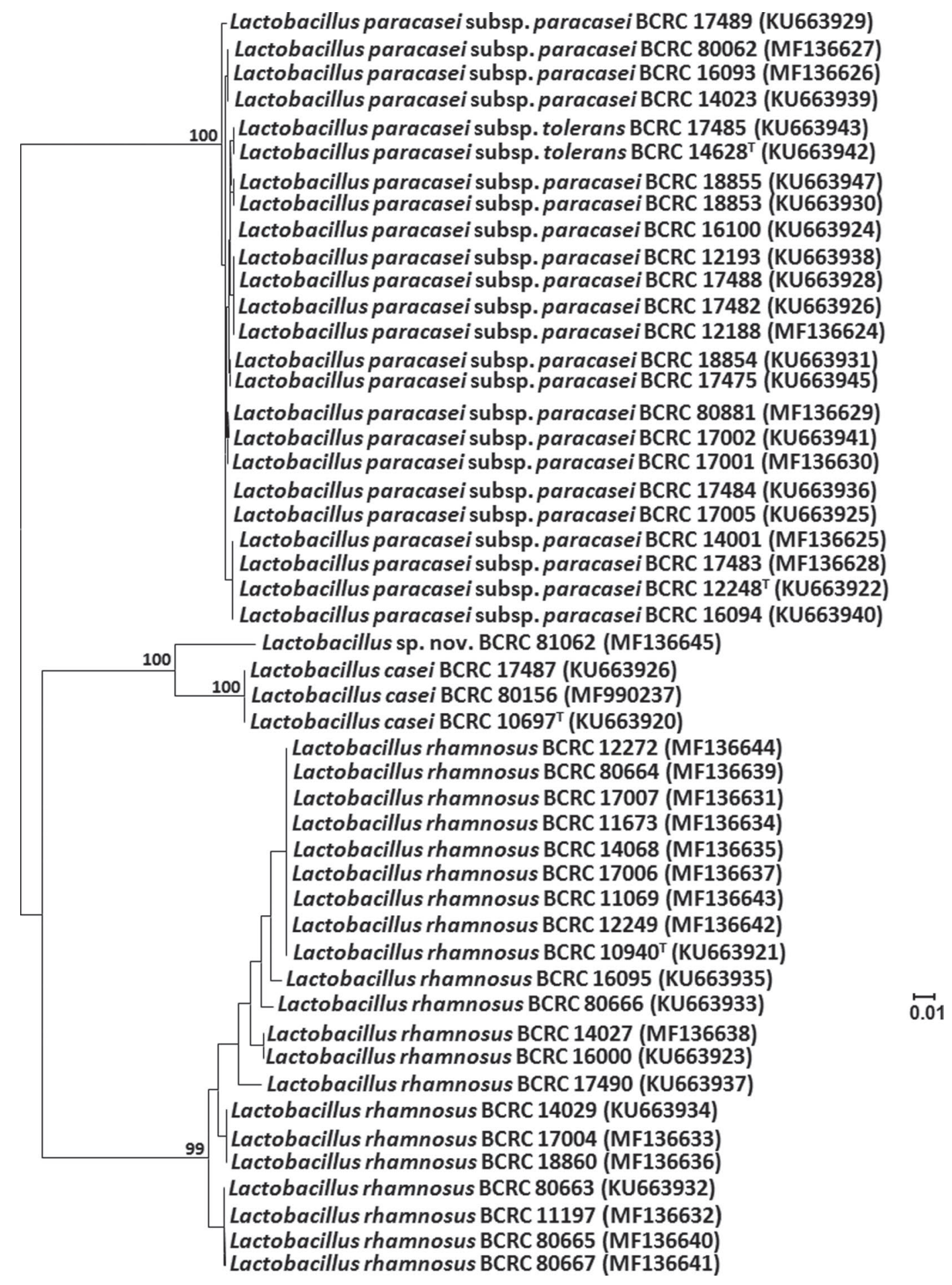

Figure 1. Phylogenetic tree of the Lactobacillus casei group strains based on their mutL gene sequences. The tree was constructed using the neighbor-joining method. Only bootstrap percentages above $80 \%$ are indicated (based on 1,000 replications). The scale bar represents $0.01 \%$ sequence divergence. BCRC = Bioresource Collection and Research Center at Food Industry Research and Development Institute (Hsinchu, Taiwan). 
L.casei BCRC 10697

L. rhamnosus BCRC 10940

L.paracasei BCRC 12248

L.Casei BCRC 10697

L. rhamnosus BCRC 10940

L.paracasei BCRC 12248

L.casei BCRC 10697

L. rhamnosus BCRC 10940

L.paracase i BCRC 12248

L.casei BCRC 10697

L. rhamnosus BCRC 10940

L.paracasei BCRC 12248

L. Case i BCRC 10697

L. rhamnosus BCRC 10940

L.paracase i BCRC 12248

L. casei BCRC 10697

L. rhamnosus BCRC 10940

L.paracasei BCRC 12248

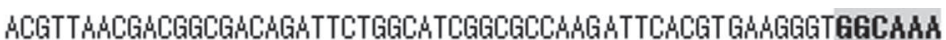

spLcasei-mutlF $\rightarrow$

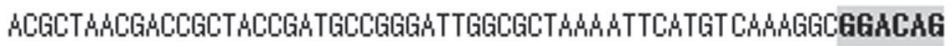

spLrham-mutlF $\rightarrow$

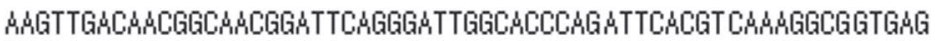

GTCGAGATCAACTACGGCGGCGCATCGCGGGGCACGGATGTTGAAGTGTCTGATCTG

GTAGAHATCAHCACAGCGGCGCATCGTCGGGACCGATGTCGARTGAGCGATCTG

GTTGA\&GCGCATCGACAGCGCTCACCGACGCGGTACGGATGTTG\&AGTA\&TGATTT

TTCTTCA ACACCAGCGCGCCTGA

TTTTTTABCACTCCAGCACGGCTGA\&GTACATGA\&GTCGCABCAGACCGACTTGGCA\&G

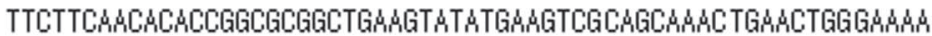

АTTGTCGATATCGTTAGTCGGCTGGCCTTGGCTASTCCCAGCATTECTT TTACETETCG

$\leftarrow$ spLcasei-mutlR

ATTGTTGATATTGTCAGTCGGCTGGCATTAGCTABCCCTAAGATTEGTTIACETCAEC

$\leftarrow$ spLrham-mutlR

ATTGTGGATATCGTCAGCCG\&TTGGCG\&TCGCA\&\&TCCTEATATTECCTTCACETTICA

spLpara-mutlF $\rightarrow$

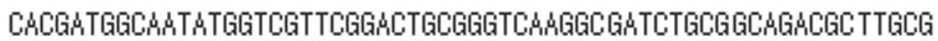

CATGACGGCA A ATGATGGTGCGGACAGCCGGCA\&GGTGATCT\&CGCCAGACAT TGGCG

САTG\&TGGCA A TGGTGGTGGCACAGCTGGTCA \&GTGATTGCGCCAGACGCTGGCC

GGAнTCTATGGCTTGCCAGT\&GCGCGGTCGATGGTTGACTTTC\&GGCGCAGGATCTTGAT

GGCATTTACGGTTTGCCGGTTGCTCGGTCA TGGTCGACTTTCABGCGAGGATCTTGAT

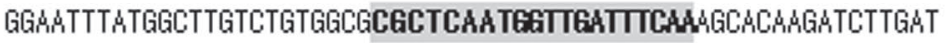

$\leftarrow$ spLpara-mutlR

Figure 2. Multiple sequence alignment was performed on mutL sequences of Lactobacillus casei group members. Regions representing the sequences of species-specific primers are shaded. L. = Lactobacillus; BCRC = Bioresource Collection and Research Center at Food Industry Research and Development Institute (Hsinchu, Taiwan). 
between 2.00 and 2.29 could be reliably identified at the genus and probably the species levels. In addition, cluster analysis was performed to construct the MSP dendrogram by using the standard settings of MALDI Biotyper 3.1 (Bruker Daltronics GmbH).

\section{ClinProTools Statistical Analysis}

The $L$. paracasei strains (including 2 subspecies) were further analyzed using ClinProTools software version 3.0. The imported raw spectra of each investigated strain were automatically recalibrated and normalized so that statistical analyses such as principal component analysis and the application of classification models could be performed. A 3-dimensional principal component analysis score plot was displayed, and a supervised neural network model was generated based on the discriminating peaks. In addition, the recognition capability and cross-validation of the model were calculated to demonstrate its reliability and accuracy.

\section{Isolation and Identification of LCG-Related Strains from Probiotic Products}

Several probiotic samples (yogurt drinks, probiotic powders and capsules) of different brands were collected. The LCG-related strains were isolated using serial dilution and plating methods and were identified by MALDI-TOF MS and species-specific PCR assays.

\section{RESULTS AND DISCUSSION}

Sequencing technology has continued to advance; genome-wide comparisons have been enabled by the use of next-generation sequencing and have been used for taxonomic assignments and applied phylogeny estimation within the LCG (Toh et al., 2013). However, whole-genome sequencing-based methods are still difficult to routinely use in general laboratories. In contrast, comparing the sequences of protein-encoding genes is an alternative method to whole-genome sequencing, and the use of these sequences as molecular markers in microbial classification and identification has increased. The mutL gene encodes a DNA mismatch repair protein that plays a critical role in maintaining genomic stability by correcting DNA replication errors (Li, 2008). The mutL gene has been used as a multilocus sequence typing target for the differentiation of $L$. casei, L. paracasei, and L. rhamnosus strains (Parolo et al., 2011; Bao et al., 2016; Poluektova et al., 2017). In this study, a degenerate primer pair (Lcagp-mutlF3/ R4) was used to successfully amplify a PCR amplicon consisting of approximately $800 \mathrm{bp}$ of the mutL gene from LCG strains; this product was then used for sequencing. The average sequence identity of the mutL gene among the LCG type strains was $78.8 \%$, which was significantly lower than that of the $16 \mathrm{~S}$ rRNA gene (98.9\%) and other common housekeeping genes such as $d n a K(85.2 \%)$, dnaJ (83.2\%), hsp60 (88.4\%), rpoA (95.1\%), tuf (93.6\%), and pheS (82.6\%) and was comparable with that of other high-resolution DNA targets such as yycH (78.5\%) and recA $(74.0 \%$; Felis et al., 2001; Naser et al., 2007; Huang and Lee, 2011; Yu et al., 2012; Huang et al., 2014, 2015). The mutL gene could therefore serve as a valuable taxonomic marker and contribute to the current multilocus sequence analysis scheme used to characterize the LCG. Nevertheless, $L$. paracasei subspecies strains could not be discriminated by these housekeeping genes but could be differentiated based on their phenotypic characteristics (Collins et al., 1989).

We found one strain (Lactobacillus sp. BCRC 81062) in which the mutL sequence had low similarity with those of the members of LCG $(78.8-90.4 \%)$ but in which 16S rRNA gene similarity was very high (99.0$99.3 \%$ ). The topology of the neighbor-joined mutL tree was clearly separated into clusters of different species, and the nodes were supported by high bootstrap values (>80\%; Figure 1). The strain BCRC 81062 was found in a separate cluster close to L. casei. Furthermore,

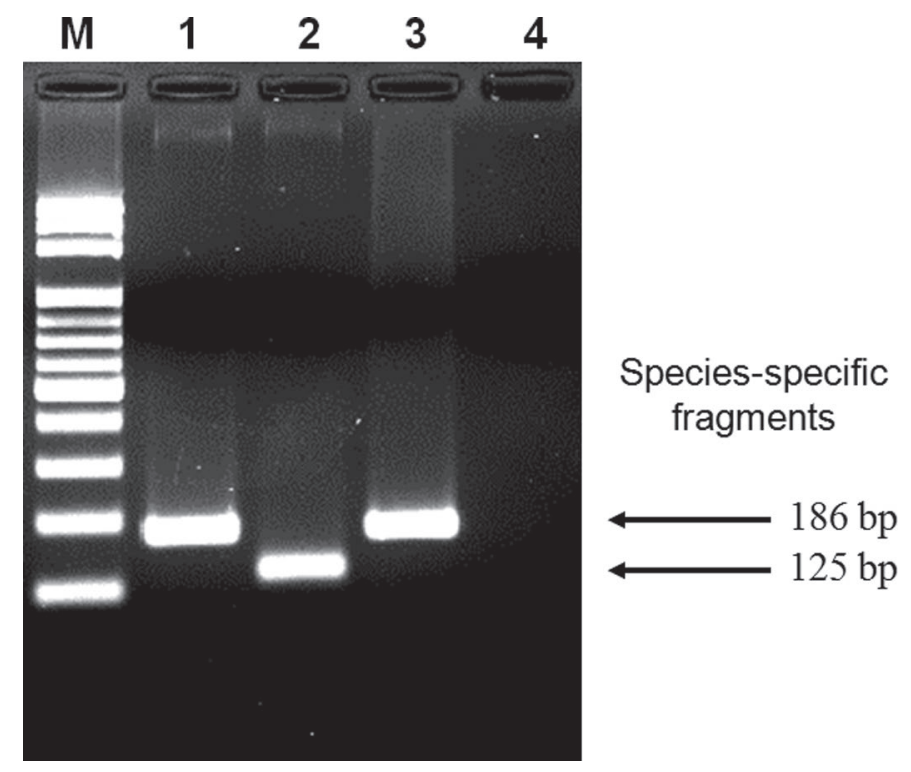

Figure 3. Gel electrophoresis of PCR products amplified using species-specific primers. M: 100-bp DNA ladder markers. Lanes: (1) Lactobacillus casei BCRC $10697^{\mathrm{T}}$, (2) Lactobacillus paracasei $\mathrm{ssp}$. paracasei $12248^{\mathrm{T}}$, (3) Lactobacillus rhamnosus BCRC $10940^{\mathrm{T}}$, and (4) negative control. BCRC $=$ Bioresource Collection and Research Center at Food Industry Research and Development Institute (Hsinchu, Taiwan). 
all mutL gene sequences were deposited in GenBank (Supplemental Table S1; https://doi.org/10.3168/ jds.2017-13642), and this accumulated sequence data could be used to develop specific PCR primers and directly identify particular LCG species (Huang et al., 2014). Three pairs of primers, each targeting a different species in the LCG, were designed based on multiple sequence alignments of the mutL gene (Figure 2), and these primers successfully generated a single speciesspecific band $(186,125$, and $186 \mathrm{bp})$ when used in PCR reactions with DNA from reference strains of $L$. casei, L. paracasei, and L. rhamnosus DNA, respectively (Figure 3; Table 1). Only BCRC 81062 and other nontarget Lactobacillus strains failed to amplify a species-specific amplicon.
Commercial MALDI-TOF MS databases are currently largely developed for clinical diagnosis (Croxatto et al., 2012; Murray, 2012; Starostin et al., 2015; Angeletti, 2017). A case in which an LCG-related probiotic product was misidentified by interpreting MALDI-TOF MS results using a Bruker database has been reported (Angelakis et al., 2011). Database and spectrum quality are the key factors in determining the accuracy of MALDI-TOF MS identification. Hence, it is essential to use entries representing most phylogenetic taxa within a closely related species group. In this study, all type strains of the LCG were used to build an IHDB under optimized culture conditions (cultivated anaerobically on De Man, Rogosa and Sharpe agar for $20 \mathrm{~h}$ at $37^{\circ} \mathrm{C}$ ). The proteomic patterns of each type strain

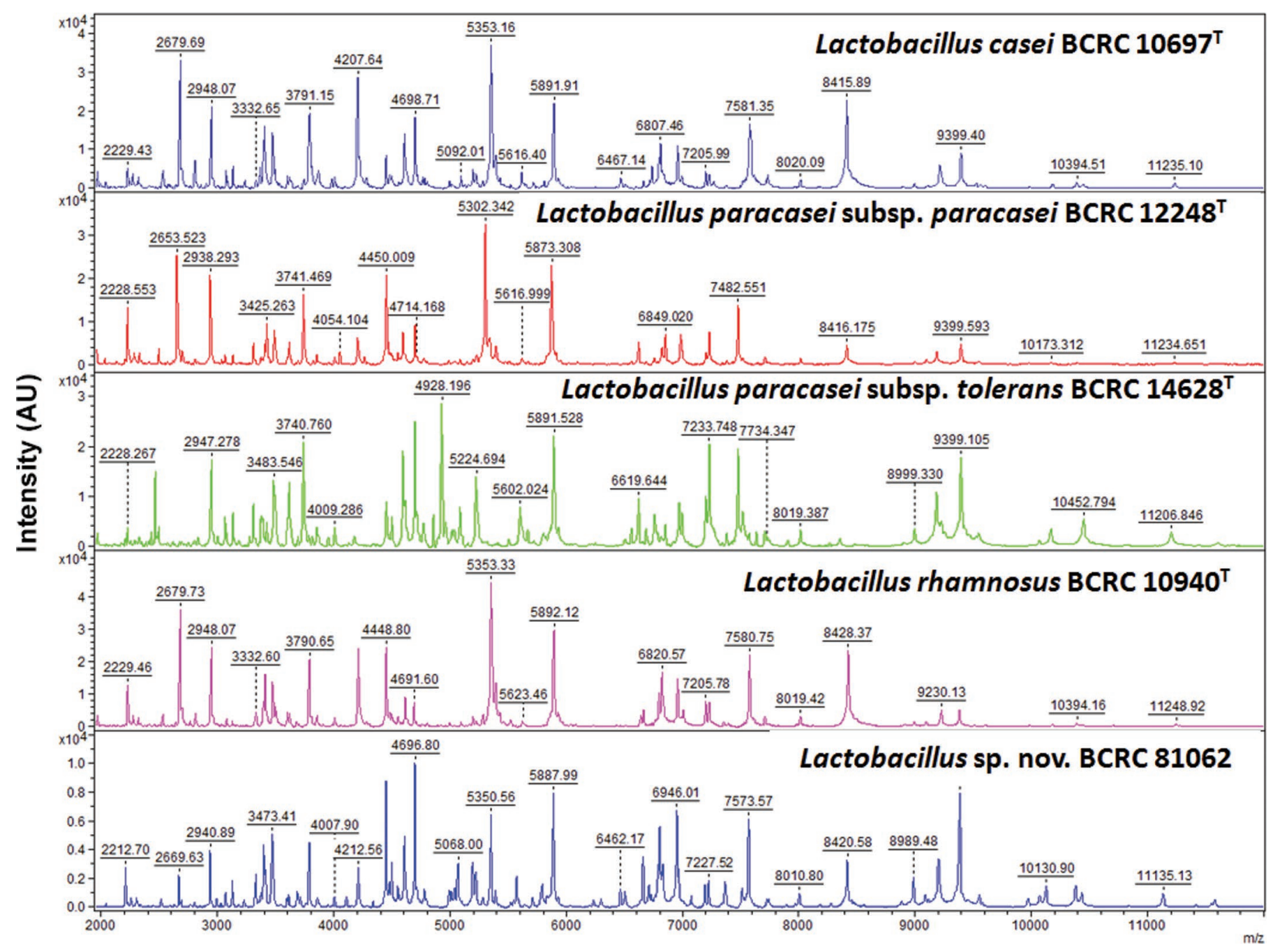

Figure 4. Representative spectra from 4 Lactobacillus casei group type strains in the in-house database supplemented by matrix-assisted laser desorption/ionization time-of-flight mass spectrometry data. AU $=$ arbitrary units; $m / z=$ mass-to-charge ratio. $\mathrm{BCRC}=\mathrm{Bioresource}$ Collection and Research Center at Food Industry Research and Development Institute (Hsinchu, Taiwan). Color version available online. 
contained unique peaks (Figure 4) and might serve as potential biomarkers for further differentiation. A total of 48 replicates for each strain were performed, and the obtained spectra were analyzed and screened using FlexAnalysis software. To determine the relationships among replicates, the CCI indicators between spectra were calculated. High CCI values (near 1) indicated almost identical spectra (data not shown). At least 20 good-quality spectra of each strain were loaded into a database and used for MSP creation with MALDI Biotyper software. We attempted to blindly reidentify each strain to determine the reproducibility of the IHDB. All LCG type strains that were already present in the IHDB were correctly identified, with scores ranging from $2.63 \pm 0.02$ to $2.65 \pm 0.03$. For subsequent validation of the IHDB, 44 LCG isolates whose identities had been confirmed on the basis of their DNA sequences were used for evaluation. The results showed that most isolates were correctly identified to the species level with high score values $(2.45 \pm 0.1$; Table 1$)$ except for strain BCRC 81062, which was identified as L. casei with a relatively low score $(2.02 \pm 0.02)$.

Biomarkers in bacteria are mainly ribosomal proteins and include a few housekeeping proteins, which is comparable with the multilocus sequence analysis of the housekeeping genes used to determine higher level taxonomy and phylogeny (Welker and Moore, 2011). In recent years, a ribosomal proteins typing-based method using MALDI-TOF MS has been proposed and successfully differentiated Bacillus spp., Bifidobacterium animalis, Bifidobacterium longum, Lactococcus lactis, and LCG (Tanigawa et al., 2010; Hotta et al., 2011; Sato et al., 2011, 2012; Ruiz-Moyano et al., 2012). The MSP spectra of all reference strains were used in the construction of a dendrogram and revealed unambiguous clustering of 5 main groups, each defined by species, subspecies, and a candidate novel taxon (Figure 5), which showed good concordance with the phylogenetic tree based on mut $L$ gene sequences, except for the $L$. paracasei subspecies. To identify the potential biomarkers of $L$. paracasei subspecies, we performed a statistical analysis using ClinProTools software. The resulting distribution map clearly displayed that $L$. $p a-$ racasei strains could be differentiated into 2 categories (Figure 6a), and a supervised neural network model was constructed based on 2 peaks (mass-to-charge ratio 4,930 and 5,303; Figure 6b); the model resulted in recognition capability and cross-validation values of $100 \%$. The specificity of this model was tested $(\mathrm{n}=24)$, and it demonstrated 100\% accuracy (Table 2). However,

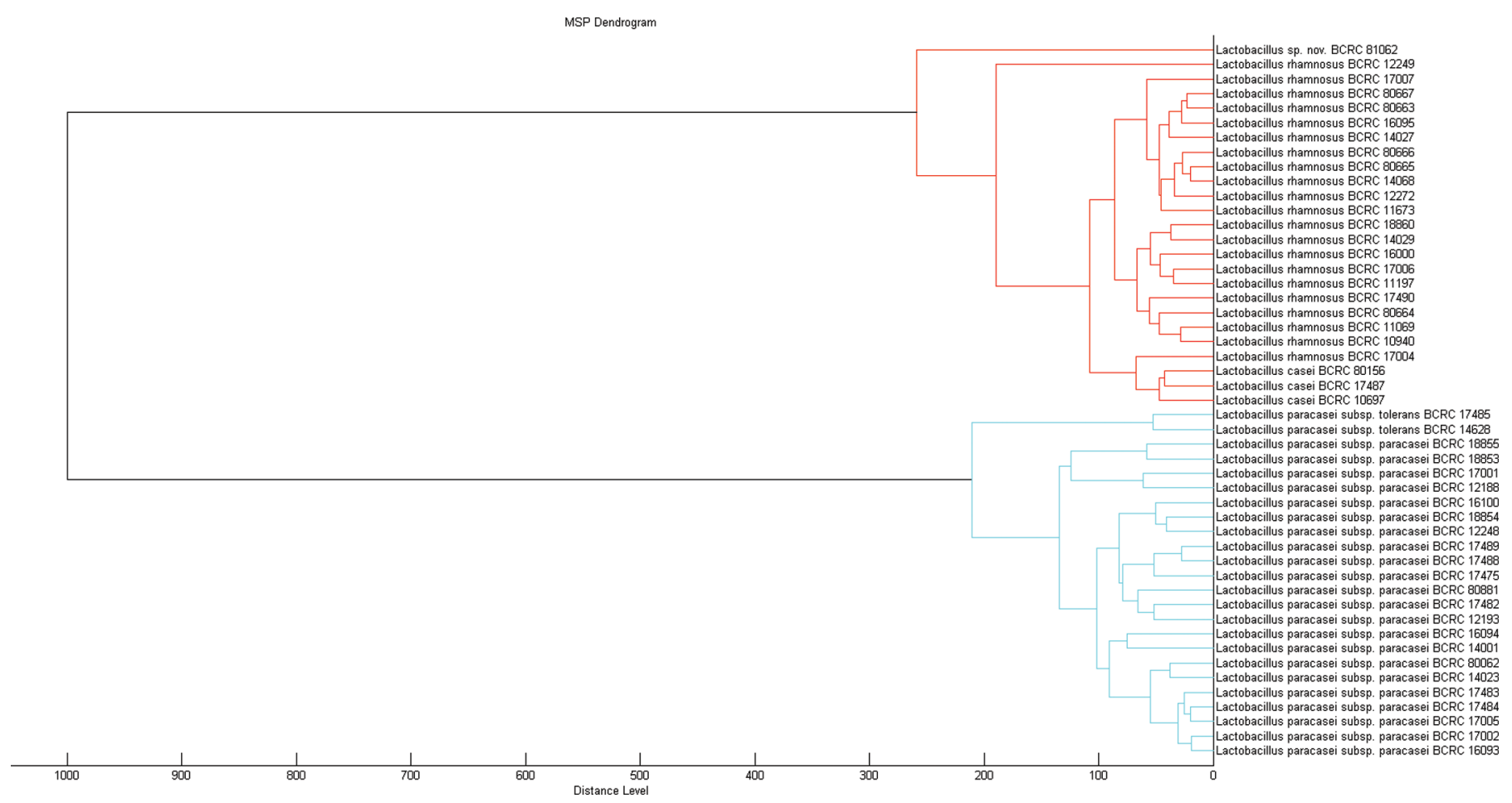

Figure 5. Dendrogram showing the clustering of the 49 Lactobacillus casei group strains based on matrix-assisted laser desorption/ionization time-of-flight mass spectrometry analysis. BCRC $=$ Bioresource Collection and Research Center at Food Industry Research and Development Institute (Hsinchu, Taiwan). MSP = main spectrum profile. Color version available online. 
Table 2. Classification results of the subspecies of Lactobacillus paracasei strains using statistical analysis in ClinProTools software (Bruker Daltonics, Billerica, MA)

\begin{tabular}{llcc}
\hline & & \multicolumn{2}{c}{ Discriminatory peaks $(m / z)^{1}$} \\
\cline { 3 - 4 } L. paracasei subspecies & Class & 4,930 & 5,303 \\
\hline paracasei $($ no. $=22)$ & 1 & - & + \\
tolerans $($ no. $=2)$ & 2 & + & - \\
\hline
\end{tabular}

${ }^{1}$ Biomarker peaks were screened by the supervised neural network model. $m / z=$ mass-to-charge ratio. $+=$ peak present; $-=$ no peak found.

these 2 peaks could not be identified due to the low abundance and instrumental limitations. Subsequently, 7 strains isolated from different probiotic samples that claimed to contain viable LCG-related species were analyzed using MALDI-TOF MS plus ClinProTools and species-specific PCR. These lactic acid bacteria were identified as L. casei, L. paracasei $\mathrm{ssp}$. paracasei, and $L$. rhamnosus, respectively (Table 3 ). However, we found a discrepancy between the labeled species and the actual identified result in probiotic capsule products.

The current method for the confirmation of closely related prokaryotic taxa involves 2 steps: 16S rRNA sequencing is first used to assign an unknown strain at the species or species-group level, which then defines the genes and primers to be used to assign the strain to the species or subspecies level (Gevers et al., 2005). A 2-tier strategy has been successfully applied to distinguish many phylogenetically closely related bacteria,

\section{(a)}

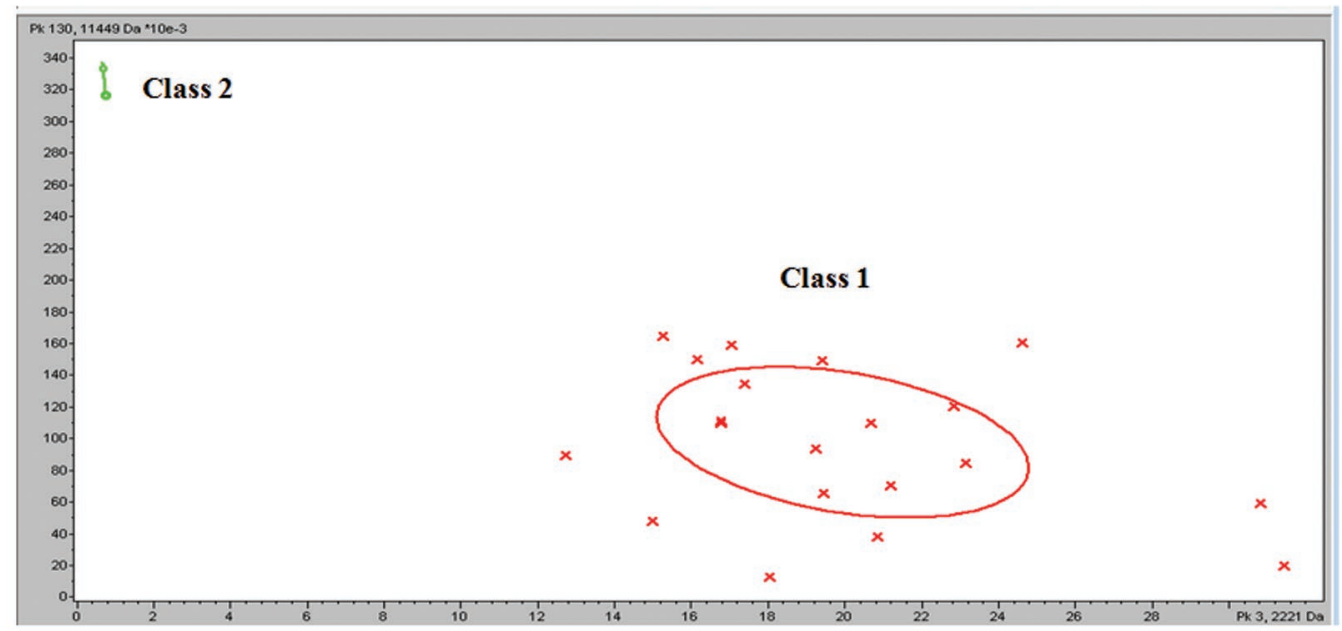

(b)

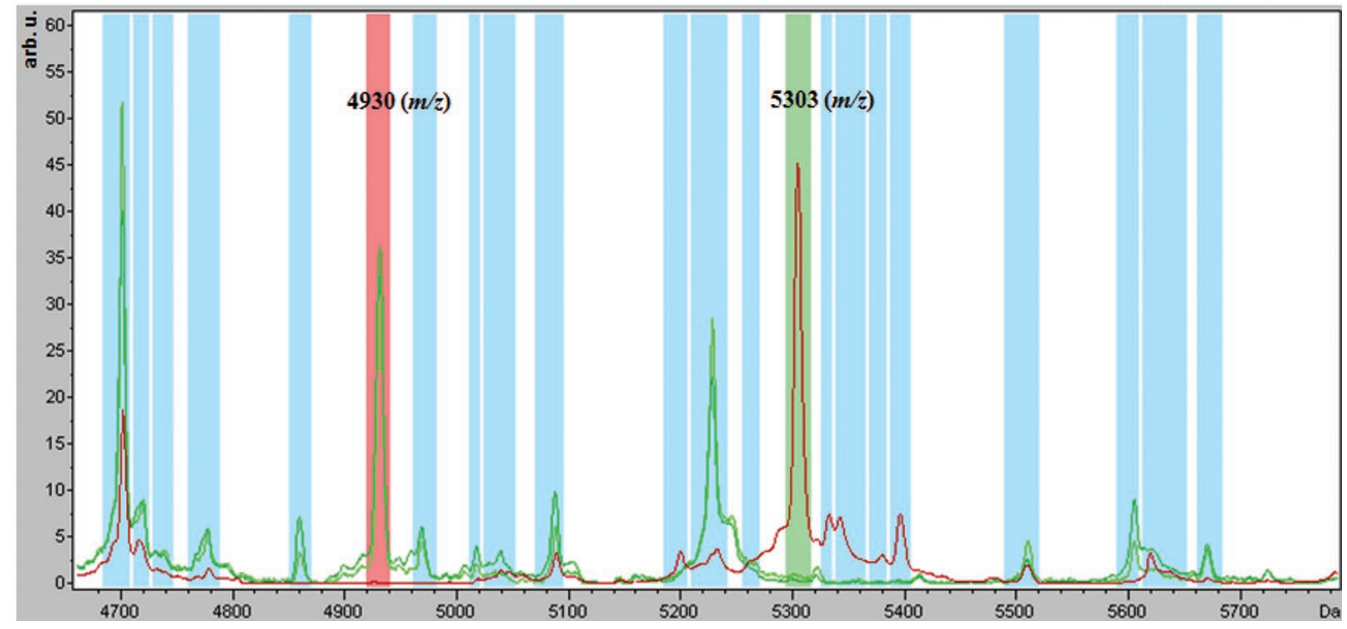

Figure 6. ClinProTools (Billerica, MA) statistical analysis of Lactobacillus paracasei subspecies strains. (a) Two-dimensional peak distribution diagrams. Red and green denote class 1 and class 2 L. paracasei, respectively. (b) The mass peaks at mass-to-charge ratio ( $m / z) 4930$ and 5303 in L. paracasei ssp. tolerans and L. paracasei ssp. paracasei strains, respectively. Color version available online. 
Table 3. Matrix-assisted laser desorption/ionization time-of-flight (MALDI-TOF) MS and species-specific PCR identification of Lactobacillus casei group-related strains in commercial probiotic products

\begin{tabular}{|c|c|c|c|c|c|}
\hline \multirow[b]{2}{*}{ Probiotic product } & \multirow[b]{2}{*}{ Label species } & \multirow[b]{2}{*}{ MALDI-TOF MS } & \multicolumn{3}{|c|}{ Species-specific $\mathrm{PCR}^{1}$} \\
\hline & & & Lcas $^{2}$ & Lparacas $^{3}$ & Lrham $^{4}$ \\
\hline Yogurt drink 2 & L. paracasei & L. paracasei ssp. paracasei & - & + & - \\
\hline Probiotic powder 1 & Lactobacillus casei & L. casei & + & - & - \\
\hline Probiotic powder 2 & L. casei & L. casei & + & - & - \\
\hline Probiotic powder 4 & L. rhamnosus & L. rhamnosus & - & - & + \\
\hline Probiotic capsule & L. casei $^{5}$ & L. rhamnosus & - & - & + \\
\hline
\end{tabular}

${ }^{1}+=$ PCR amplicons with each primer pair detected; - = PCR amplicons with each primer pair not detected.

${ }^{2}$ Species-specific PCR amplification with primer spLcasei-mutlF $\left(5^{\prime}\right.$-GGCAAAGTCGAGGATCAAACTA-3')/-R (5'-CGACACCGTAAAAGCAATGCTG-3').

${ }^{3}$ Species-specific PCR amplification with primer spLpara-mutlF (5'-GATATTGCCTTCACGGTTTCAC-3')/-R (5'-TTGAARTCAACCATTGAGCG-3').

${ }^{4}$ Species-specific PCR amplification with primer spLrham-mutlF (5'-GGRCAGGTAGAAAGTCAAACGA-3')/-R (5'-GCTGACCGTAAACGCAATCTTAG-3').

${ }^{5}$ Discordance between the labeled and identified strains.

such as members of the Lactobacillus acidophilus group, the L. casei group, the Lactobacillus plantarum group, and the Lactobacillus sakei group (Naser et al., 2007; Huang et al., 2010, 2012, 2014). The MALDI-TOF MS ClinProTools method provides higher resolution (down to the subspecies level), faster analysis ( $2 \mathrm{~h}$ after the culture), and lower reagent costs (around $\$ 0.20$ /sample) than mutL gene sequencing and species-specific PCR assays. Consequently, the MALDI-TOF MS ClinProTools method developed in this study is preferable.

\section{CONCLUSIONS}

Our data confirm that proteomic identification within the LCG, based on combining the use of an IHDB and the application of ClinProTools to MALDI-TOF MS data, showed superior discrimination power and required less analysis time than DNA sequencing and PCR-based methods did and can be applied to achieve efficient quality control of probiotic products. According to the preliminary sequence- and proteomics-based data, we deduced that BCRC 81062 may represent an undescribed new taxon, and we will integrate a polyphasic taxonomic strategy to describe novel species in the future.

\section{ACKNOWLEDGMENTS}

This research was supported by Ministry of Science and Technology, Republic of China (project no. MOST 106-2320-B-080-001) and Ministry of Economic Affairs, Republic of China (project no. 106-EC-17-A-22-0525).

\section{REFERENCES}

Ahrne, S., G. Molin, and S. Stahl. 1989. Plasmids in Lactobacillus strains isolated from meat and meat products. Syst. Appl. Microbiol. 11:320-325.

Angelakis, E., M. Million, M. Henry, and D. Raoult. 2011. Rapid and accurate bacterial identification in probiotics and yoghurts by MALDI-TOF mass spectrometry. J. Food Sci. 76:M568-M572.

Angeletti, S. 2017. Matrix assisted laser desorption time of flight mass spectrometry (MALDI-TOF MS) in clinical microbiology. J. Microbiol. Methods 138:20-29.

Ashraf, R., and N. P. Shah. 2014. Immune system stimulation by probiotic microorganisms. Crit. Rev. Food Sci. Nutr. 54:938-956.

Bao, Q., Y. Song, H. Xu, J. Yu, W. Zhang, B. Menghe, H. Zhang, and Z. Sun. 2016. Multilocus sequence typing of Lactobacillus casei isolates from naturally fermented foods in China and Mongolia. J. Dairy Sci. 99:5202-5213.

Bizzini, A., and G. Greub. 2010. Matrix-assisted laser desorption ionization time-of-flight mass spectrometry, a revolution in clinical microbial identification. Clin. Microbiol. Infect. 16:1614-1619.

Campanella, J. J., L. Bitincka, and J. Smalley. 2003. MatGAT: An application that generates similarity/identity matrices using protein or DNA sequences. BMC Bioinformatics 4:29.

Chen, H., C. K. Lim, Y. K. Lee, and Y. N. Chan. 2000. Comparative analysis of the genes encoding $23 \mathrm{~S}-5 \mathrm{~S}$ rRNA intergenic spacer regions of Lactobacillus casei-related strains. Int. J. Syst. Evol. Microbiol. 50:471-478.

Collins, M. D., B. A. Phillips, and P. Zanoni. 1989. Deoxyribonucleic acid homology studies of Lactobacillus casei, Lactobacillus paracasei sp. nov., ssp. paracasei and ssp. tolerans, and Lactobacillus rhamnosus sp. nov., comb. nov. Int. J. Syst. Bacteriol. 39:105-108.

Croxatto, A., G. Prodhom, and G. Greub. 2012. Applications of MALDI-TOF mass spectrometry in clinical diagnostic microbiology. FEMS Microbiol. Rev. 36:380-407.

Dellaglio, F., L. M. T. Dicks, M. Dutoit, and S. Torriani. 1991. Designation of ATCC 334 in place of ATCC 393 (NCDO 161) as the neotype strain of Lactobacillus casei ssp. casei and rejection of the name Lactobacillus paracasei. Int. J. Syst. Bacteriol. 41:340-342.

Dellaglio, F., G. E. Felis, and S. Torriani. 2002. The status of the species Lactobacillus casei (Orla-Jensen 1916) Hansen and Lessel 1971 and Lactobacillus paracasei Collins et al. 1989. Request for an opinion. Int. J. Syst. Evol. Microbiol. 52:285-287. 
Felis, G. E., F. Dellaglio, L. Mizzi, and S. Torriani. 2001. Comparative sequence analysis of a $r e c A$ gene fragment brings new evidence for a change in the taxonomy of the Lactobacillus casei group. Int. J. Syst. Evol. Microbiol. 51:2113-2117.

Felsenstein, J. 1993. PHYLIP (Phylogeny Inference Package). Version 3.5c. Department of Genetics, University of Washington, Seattle.

Gevers, D., F. M. Cohan, J. G. Lawrence, B. G. Spratt, T. Coenye, E. J. Feil, E. Stackebrandt, Y. van de Peer, P. Vandamme, F. L. Thompson, and J. Swings. 2005. Opinion: Re-evaluating prokaryotic species. Nat. Rev. Microbiol. 3:733-739.

Hotta, Y., J. Sato, H. Sato, A. Hosoda, and H. Tamura. 2011. Classification of the genus Bacillus based on MALDI-TOF MS analysis of ribosomal proteins coded in $S 10$ and spc operons. J. Agric. Food Chem. 59:5222-5230.

Huang, C. H., M. T. Chang, and L. Huang. 2014. Use of highly variable gene $(y y c H)$ as DNA marker to resolve interspecific relationships within the Lactobacillus casei group and a target for developing novel species-specific PCR primers. Eur. Food Res. Technol. 239:719-724

Huang, C. H., M. T. Chang, L. Huang, and W. S. Chu. 2015. The dnaJ gene as a molecular discriminator to differentiate among species and strain within the Lactobacillus casei group. Mol. Cell. Probes $29: 479-484$.

Huang, C. H., M. T. Chang, M. C. Huang, L. T. Wang, L. Huang, and F. L. Lee. 2012. Discrimination of the Lactobacillus acidophilus group using sequencing, species-specific PCR and SNaPshot minisequencing technology based on the recA gene. J. Sci. Food Agric. 92:2703-2708.

Huang, C. H., L. Huang, M. T. Chang, and K. L. Chen. 2016. Establishment and application of an analytical in-house database (IHDB) for rapid discrimination of Bacillus subtilis group (BSG) using whole-cell MALDI-TOF MS technology. Mol. Cell. Probes 30:312-319.

Huang, C. H., and F. L. Lee. 2011. The dnaK gene as a molecular marker for the classification and discrimination of the Lactobacillus casei group. Antonie van Leeuwenhoek 99:319-327.

Huang, C. H., F. L. Lee, and J. S. Liou. 2010. Rapid discrimination and classification of the Lactobacillus plantarum group based on a partial dnaK sequence and DNA fingerprinting techniques. Antonie van Leeuwenhoek 97:289-296.

Huys, G., M. Vancanneyt, K. D'Haene, V. Vankerckhoven, H. Goossens, and J. Swings. 2006. Accuracy of species identity of commercial bacterial cultures intended for probiotic or nutritional use. Res. Microbiol. 157:803-810.

Kim, M., H. S. Oh, S. C. Park, and J. Chum. 2014. Towards a taxonomic coherence between average nucleotide identity and 16S rRNA gene sequence similarity for species demarcation of prokaryotes. Int. J. Syst. Evol. Microbiol. 64:346-351.

Kimura, M. 1980. A simple method for estimating evolutionary rates of base substitutions through comparative studies of nucleotide sequences. J. Mol. Evol. 16:111-120.

Li, G. M. 2008. Mechanisms and functions of DNA mismatch repair. Cell Res. 18:85-98

Mattarelli, P., W. Holzapfel, C. M. Franz, A. Endo, G. E. Felis, W. Hammes, B. Pot, L. Dicks, and F. Dellaglio. 2014. Recommended minimal standards for description of new taxa of the genera Bifidobacterium, Lactobacillus and related genera. Int. J. Syst. Evol. Microbiol. 64:1434-1451.

Moore, E. R., and R. Rossello-Mora. 2011. MALDI-TOF MS: A return to phenotyping in microbial identification? Syst. Appl. Microbiol. $34: 1$.

Murray, P. R. 2012. What is new in clinical microbiology-microbial identification by MALDI-TOF mass spectrometery: A paper from the 2011 William Beaumont Hospital Symposium on molecular pathology. J. Mol. Diagn. 14:419-423.

Naser, S. M., P. Dawyndt, B. Hoste, D. Gevers, K. Vandemeulebroecke, I. Cleenwerck, M. Vancanneyt, and J. Swings. 2007. Identification of lactobacilli by pheS and rpoA gene sequence analyses. Int. J. Syst. Evol. Microbiol. 57:2777-2789.

Neville, S. A., A. Lecordier, H. Ziochos, M. J. Chater, I. B. Gosbell, M. W. Maley, and S. J. van Hall. 2011. Utility of matrix-assisted laser desorption ionization-time of flight mass spectrometry following introduction for routine laboratory bacterial identification. J. Clin. Microbiol. 49:2980-2984.

Orlando, A., M. Linsalata, and F. Russo. 2016. Antiproliferative effects on colon adenocarcinoma cells induced by co-administration of vitamin $\mathrm{K} 1$ and Lactobacillus rhamnosus GG. Int. J. Oncol. 48:2629-2638.

Parolo, C. C., T. Do, U. Henssge, L. S. Alves, F. C. de Santana Giongo, G. Corcao, M. Maltz, and D. Beighton. 2011. Genetic diversity of Lactobacillus paracasei isolated from in situ human oral biofilms. J. Appl. Microbiol. 111:105-113.

Pavlovic, M., I. Huber, R. Konrad, and U. Busch. 2013. Application of MALDI-TOF MS for the identification of food borne bacteria. Open Microbiol. J. 7:135-141.

Petti, C. A., C. R. Polage, and P. Schreckenberger. 2005. The role of $16 \mathrm{~S}$ rRNA gene sequencing in identification of microorganisms misidentified by conventional methods. J. Clin. Microbiol. 43:6123-6125.

Poluektova, E. U., R. A. Yunes, M. V. Epiphanova, V. S. Orlova, and V. N. Danilenko. 2017. The Lactobacillus rhamnosus and Lactobacillus fermentum strains from human biotopes characterized with MLST and toxin-antitoxin gene polymorphism. Arch. Microbiol. 199:683-690.

Reid, B. 2015. The growth potential for dairy probiotics. Int. Dairy J. 49:16-22

Ruiz-Moyano, S., N. Tao, M. A. Underwood, and D. A. Mills. 2012. Rapid discrimination of Bifidobacterium animalis subspecies by matrix-assisted laser desorption ionization-time of flight mass spectrometry. Food Microbiol. 30:432-437.

Samb-Ba, B., C. Mazenot, A. Gassama-Sow, G. Dubourg, H. Richet, P. Hugon, J. C. Lagier, D. Raoult, and F. Fenollar. 2014. MALDITOF identification of the human gut microbiome in people with and without diarrhea in Senegal. PLoS One 9:e87419.

Sandrin, T. R., J. E. Goldstein, and S. Schumaker. 2013. MALDI-TOF MS profiling of bacteria at the strain level: A review. Mass Spectrom. Rev. 32:188-217.

Santos, I. C., Z. L. Hildenbrand, and K. A. Schug. 2016. Applications of MALDI-TOF MS in environmental microbiology. Analyst $141: 2827-2837$.

Sato, H., K. Teramoto, Y. Ishii, K. Watanabe, and Y. Benno. 2011. Phylogenetic analysis of Bifidobacterium longum strains based on ribosomal protein profiling by matrix assisted laser desorption/ ionization time-of-flight mass spectrometry. Syst. Appl. Microbiol. 34:76-80.

Sato, H., M. Torimura, M. Kitahara, M. Ohkuma, Y. Hotta, and H. Tamura. 2012. Characterization of the Lactobacillus casei group based on the profiling of ribosomal proteins coded in S10-spcalpha operons as observed by MALDI-TOF MS. Syst. Appl. Microbiol. 35:447-454.

Shida, K., and K. Nomoto. 2013. Probiotics as efficient immunopotentiators: Translational role in cancer prevention. Indian J. Med. Res. 138:808-814.

Skerman, V. B. D., V. McGowan, and P. H. A. Sneath. 1980. Approved lists of bacterial names. Int. J. Syst. Bacteriol. 30:225-420.

Spinali, S., A. Van Belkum, R. V. Goering, V. Girard, M. Welker, M. Van Nuenen, D. H. Pincus, M. Arsac, and G. Durand. 2015. Microbial typing by matrix-assisted laser desorption ionizationtime of flight mass spectrometry: Do we need guidance for data interpretation? J. Clin. Microbiol. 53:760-765.

Stackebrandt, E., W. Frederiksen, G. M. Garrity, P. A. Grimont, P. Kampfer, M. C. Maiden, X. Nesmem, R. Rossello-Mora, J. Swings, H. G. Truper, L. Vauterin, A. C. Ward, and W. B. Whitmanm. 2002. Report of the ad hoc committee for the re-evaluation of the species definition in bacteriology. Int. J. Syst. Evol. Microbiol. $52: 1043-1047$.

Starostin, K. V., E. A. Demidov, A. V. Bryanskaya, V. M. Efimov, A. S. Rozanov, and S. E. Peltek. 2015. Identification of Bacillus strains by MALDI TOF MS using geometric approach. Sci. Rep. 5:16989.

Tanigawa, K., H. Kawabata, and K. Watanabe. 2010. Identification and typing of Lactococcus lactis by matrix-assisted laser desorp- 
tion ionization-time of flight mass spectrometry. Appl. Environ. Microbiol. 76:4055-4062.

Thompson, J. D., T. J. Gibson, F. Plewniak, F. Jeanmougin, and D. G. Higgins. 1997. The CLUSTAL X windows interface: Flexible strategies for multiple sequence alignment aided by quality analysis tools. Nucleic Acids Res. 25:4876-4882.

Tindall, B. J. 2008. The type strain of Lactobacillus casei is ATCC 393, ATCC 334 cannot serve as the type because it represents a different taxon, the name Lactobacillus paracasei and its subspecies names are not rejected and the revival of the name 'Lactobacillus zeae' contravenes Rules 51b (1) and (2) of the International Code of Nomenclature of Bacteria. Opinion 82. Int. J. Syst. Evol. Microbiol. 58:1764-1765.

Toh, H., A. Oshima, A. Nakano, M. Takahata, M. Murakami, T. Takaki, H. Nishiyam, S. Igimi, M. Hattori, and H. Morita. 2013. Genomic adaptation of the Lactobacillus casei group. PLoS One 8:e75073.
Tran, A., K. Alby, A. Kerr, M. Jones, and P. H. Gilligan. 2015. Cost savings realized by implementation of routine microbiological identification by matrix-assisted laser desorption ionization-time of flight mass spectrometry. J. Clin. Microbiol. 53:2473-2479.

Varghese, N. J., S. Mukherjee, N. Ivanova, K. T. Konstantinidis, K. Mavrommatis, N. C. Kyrpides, and A. Pati. 2015. Microbial species delineation using whole genome sequences. Nucleic Acids Res. 43:6761-6771.

Welker, M., and E. R. Moore. 2011. Applications of whole-cell matrixassisted laser-desorption/ionization time-of-flight mass spectrometry in systematic microbiology. Syst. Appl. Microbiol. 34:2-11.

Yu, J., Z. Sun, W. Liu, Q. Bao, J. Zhang, and H. Zhang. 2012. Phylogenetic study of Lactobacillus acidophilus group, L. casei group and L. plantarum group based on partial hsp60, pheS and tuf gene sequences. Eur. Food Res. Technol. 234:927-934. 